ISSN: 2059-7983

journals.iucr.org/d

\title{
Secret life of importin- $\beta$; solenoid flexibility as the key to transport through the nuclear pore
}

\section{Gualtiero Alvisi and David A. Jans}

Acta Cryst. (2016). D72, 703-704

\section{IIUCr Journals CRYSTALLOGRAPHY JOURNALS ONLINE \\ Copyright (C) International Union of Crystallography \\ Author(s) of this paper may load this reprint on their own web site or institutional repository provided that this cover page is retained. Republication of this article or its storage in electronic databases other than as specified above is not permitted without prior permission in writing from the IUCr. \\ For further information see http://journals.iucr.org/services/authorrights.html}


STRUCTURAL BIOLOGY

ISSN 2059-7983

Keywords: importin- $\beta$; solenoid flexibility; nuclear pore.
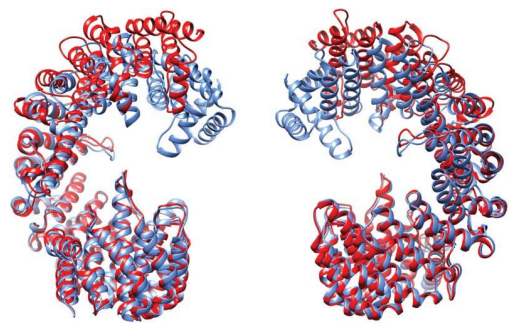

(C) 2016 International Union of Crystallography

\section{Secret life of importin- $\beta$; solenoid flexibility as the key to transport through the nuclear pore}

\author{
Gualtiero Alvisi ${ }^{\mathrm{a} *}$ and David A. Jans ${ }^{\mathrm{b} *}$ \\ ${ }^{\mathbf{a}}$ Department of Molecular Medicine, University of Padua, 35121 Padua, Italy, and ${ }^{\mathbf{b}}$ Department of Biochemistry and \\ Molecular Biology, Monash University, 3800 Clayton, Victoria, Australia. *Correspondence e-mail: \\ gualtiero.alvisi@unipd.it, david.jans@monash.edu
}

The current issue of Acta Crystallographica Section D features 'Impact of the crystallization condition on importin- $\beta$ conformation' by Tauchert et al. (2016), a significant advance in the area of nuclear transport that also has important implications for understanding the limitations of crystallization approaches. Active transport of macromolecules into and out of the eukaryotic cell nucleus occurs through the nuclear envelope (NE)-embedded multiprotein subunit nuclear pore complexes (NPCs). The transport of most cargoes is dependent on solenoid proteins belonging to the Karyopherin $\beta$ family, of which importin (Imp) $\beta 1$ is the prototype. $\operatorname{Imp} \beta 1$ is capable of recognizing specific nuclear import cargoes and transporting them across the NPC by interacting with the hydrophobic meshwork constituting the NPC core, formed by phenyl-alanine-glycine (FG) rich nucleoporins (nups). Cargo recognition can occur directly, or indirectly through adaptor proteins such as Imp $\alpha$ or snurportin, and in either case complexes are dissociated upon binding of Ran-GTP to $\operatorname{Imp} \beta 1$ on the nucleoplasmic side of the NPC. Since the discovery of $\operatorname{Imp} \beta 1$ (Görlich et al., 1995), structural studies have helped elucidate many aspects of the molecular details of cargo and adaptor binding/release (Christie et al., 2016). Unanswered questions, however, include how $\operatorname{Imp} \beta$ s achieve cargo transport across the NPC through interaction with nups (Liu \& Stewart, 2005; Bayliss et al., 2000); Tauchert et al. provide an important, new slant on this question.

$\operatorname{Imp} \beta 1$ binds to a plethora of different proteins, including cargoes, adaptors, RanGTP and nups. Previous studies have shown $\operatorname{Imp} \beta 1$ to be a solenoid formed by 19 HEAT repeats, each of which comprises two antiparallel helices connected by a turn (Cingolani et al., 1999); HEAT repeats are connected by short linkers and arranged in a superhelix, with very few long distance intraprotein interactions, enabling $\operatorname{Imp} \beta 1$ to undergo extensive changes in tertiary structure (overall protein shape), without alteration to secondary structure (HEAT repeats). Consistent with this idea, the comparison of $\operatorname{Imp} \beta$ crystal structures to date reveals a wide range of conformations varying from very compact, heart-like structures (the 'apo' or nup-bound form) to more relaxed ones (e.g. bound to RanGTP).

This observed structural variability has been postulated to be the direct effect of the binding of different partners to $\operatorname{Imp} \beta 1$ causing/inducing changes in $\operatorname{Imp} \beta 1$ folding, but $\mathrm{X}$-ray scattering (SAXS) data shows that $\operatorname{Imp} \beta 1$ alone is more relaxed in solution than in crystal lattices (Fukuhara et al., 2004). Further, molecular dynamics (MD) simulations suggest that the apo form of $\operatorname{Imp} \beta 1$ undergoes remarkable conformational changes in solution, adopting a more extended S-shaped conformation that is quite distinct to that observed in its crystalline form (Zachariae \& Grubmüller, 2008; Forwood et al., 2010). Significantly, two drastically different conformations of $\operatorname{Imp} \beta 1$ bound to the snurportin $\operatorname{Imp} \beta 1$ binding domain (IBB) were recently observed in the same crystallographic asymmetric unit (Bhardwaj \& Cingolani, 2010); this both highlights $\operatorname{Imp} \beta 1$ flexibility, and importantly underlines the fact that structures obtained in crystals may not reflect the wide range of $\operatorname{Imp} \beta 1$ conformations in solution. Forwood et al. (2010) used crystallography/SAXS/MD to show that $\operatorname{Imp} \beta 1$ assumes various different conformations in solution, postulated to result from cumulative incremental structural changes along the entire length of the solenoid, and speculated to be integral to $\operatorname{Imp} \beta 1$ 's ability to traverse the highly hydrophobic channel of the NPC. Indeed, MD simulations reveal that $\operatorname{Imp} \beta 1$ in water is extremely different from that in $50 \%$ 2,2,2-trifluoroethanol, where $\operatorname{Imp} \beta 1$ rapidly becomes more compact (Yoshimura et al., 2014); similar results have been 


\section{scientific commentaries}
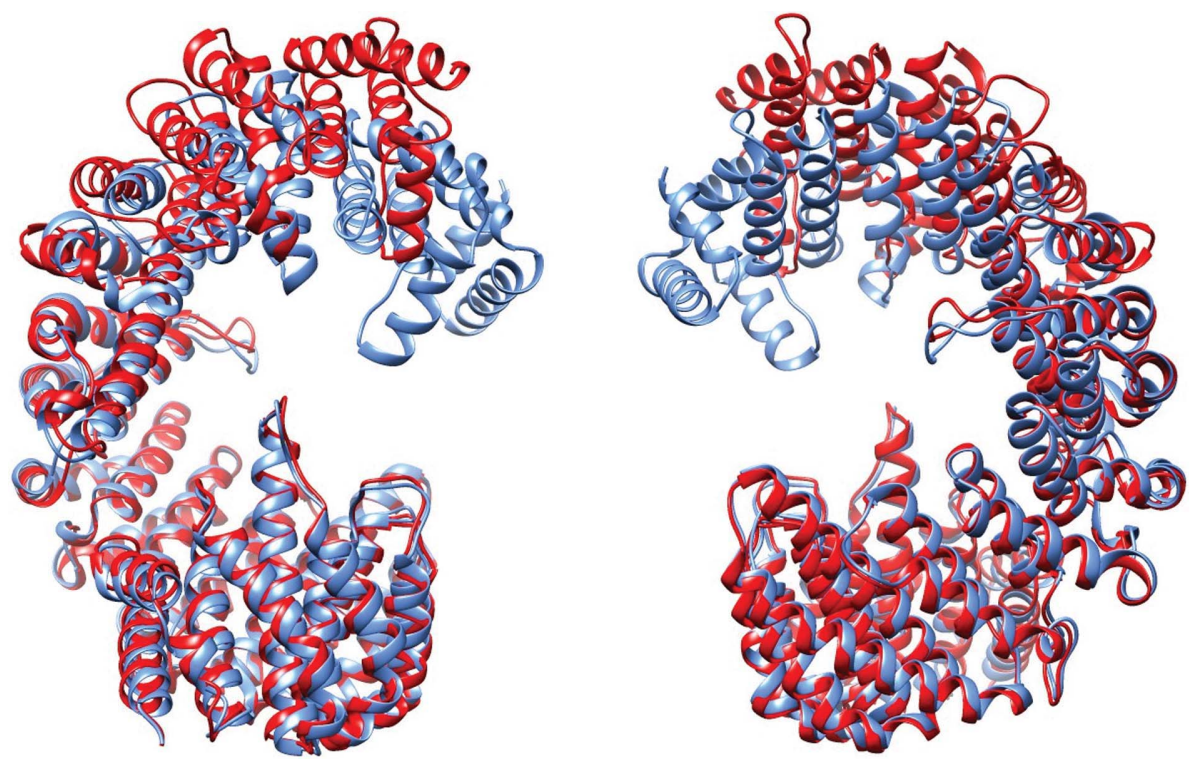

Figure 1

Effect of solvent on C. Thermophilum $\operatorname{Imp} \beta 1$ structure. The structures of $\operatorname{Imp} \beta 1$ obtained after PEG precipitation (red) or $\left(\mathrm{NH}_{4}\right)_{2} \mathrm{SO}_{4}$ (blue) precipitation are superimposed. The structure shown on the right is rotated $180^{\circ} \mathrm{C}$ with respect to that on the left.

obtained with both IBB-complexed and free $\operatorname{Imp} \beta 1$ in water compared to in methanol (Halder et al., 2015). This ability to undergo conformational changes appears to be the key to Imp $\beta 1$-mediated transport across the NPC, since crosslinking to impair this flexibility impedes nuclear translocation (Yoshimura et al., 2014).

Tauchert et al. extend these findings, proffering an interesting alternative point of view regarding the forces determining different conformations of $\operatorname{Imp} \beta 1$ in crystalline form according to the hydrophobicity of the milieu. Tauchert et al. solve the structure of $\operatorname{Imp} \beta 1$ from the thermophilic fungus Chaetomium thermophilum in two physicochemically different conditions, taking advantage of the serendipitous S107P/ V134A mutant which crystallized in the presence of the hydrophilic inorganic salt $\left(\mathrm{NH}_{4}\right)_{2} \mathrm{SO}_{4}$, adopting a much more compact structure than that of its wild-type counterpart crystallized in the presence of PEG (Fig. 1). These findings are confirmed in solution using SAXS, the important overall implication being that solvent hydrophobicity strongly affects $\operatorname{Imp} \beta 1$ conformation, and hence can be of key importance in the dominant conformation crystallized. Further, analyzing previous $\operatorname{Imp} \beta 1$ crystal structures, the authors find a strong correlation between the extent to which $\operatorname{Imp} \beta 1$ takes an extended conformation, and the amount of PEG in the buffer. Importantly, analysis of Imp $\alpha$ crystal structure pairs solved in either PEG or $\left(\mathrm{NH}_{4}\right)_{2} \mathrm{SO}_{4}$ indicates that these properties do not apply, since $\operatorname{Imp} \alpha$, although structurally related to $\operatorname{Imp} \beta 1$, is less flexible. The polar/apolar regions of PEG would appear to mimic nup FG repeats within the NPC, suggesting that $\operatorname{Imp} \beta 1$ traverses the NPC in an extended conformation, in contrast to what has been proposed previously (Halder et al., 2015; Yoshimura et al., 2014). The only crystal structures obtained so far between $\operatorname{Imp} \beta 1$ and NPC components used short FG-rich nup fragments, and thus do not shed light on the state of $\operatorname{Imp} \beta 1$-nups interaction within the core of the NPC (Liu \& Stewart, 2005; Bayliss et al., 2000). Importantly, apart from giving an important new insight into this aspect of nuclear transport, Tauchert et al.'s study clearly underlines the need for more extensive $\operatorname{Imp} \beta 1$-nup complex structures, with the proviso that the buffer systems used have to be considered critically (with a grain of salt perhaps?), and ideally should also be analysed in detail using complementary approaches such as SAXS.

\section{References}

Bayliss, R., Littlewood, T. \& Stewart, M. (2000). Cell, 102, 99-108. Bhardwaj, A. \& Cingolani, G. (2010). Biochemistry, 49, 5042-5047.

Christie, M., Chang, C. W., Róna, G., Smith, K. M., Stewart, A. G., Takeda, A. A., Fontes, M. R., Stewart, M., Vértessy, B. G., Forwood, J. K. \& Kobe, B. (2016). J. Mol. Biol. 428, 2060-2090.

Cingolani, G., Petosa, C., Weis, K. \& Müller, C. W. (1999). Nature, 399, 221-229.

Forwood, J. K., Lange, A., Zachariae, U., Marfori, M., Preast, C., Grubmüller, H., Stewart, M., Corbett, A. H. \& Kobe, B. (2010). Structure, 18, 1171-1183.

Fukuhara, N., Fernandez, E., Ebert, J., Conti, E. \& Svergun, D. (2004). J. Biol. Chem. 279, 2176-2181.

Görlich, D., Kostka, S., Kraft, R., Dingwall, C., Laskey, R. A., Hartmann, E. \& Prehn, S. (1995). Curr. Biol. 5, 383-392.

Halder, K., Dölker, N., Van, Q., Gregor, I., Dickmanns, A., Baade, I., Kehlenbach, R. H., Ficner, R., Enderlein, J., Grubmüller, H. \& Neumann, H. (2015). Biophys. J. 109, 277-286.

Liu, S. M. \& Stewart, M. (2005). J. Mol. Biol. 349, 515-525.

Yoshimura, S. H., Kumeta, M. \& Takeyasu, K. (2014). Structure, 22, 1699-1710.

Tauchert, M. J., Hémonnot, C., Neumann, P., Köster, S., Ficner, R. \& Dickmanns, A. (2016). Acta Cryst. D72, 705-717.

Zachariae, U. \& Grubmüller, H. (2008). Structure, 16, 906-915. 